\title{
Routine preparation of seminal fluid specimens for transmission electron microscopy
}

\author{
TA RYDER, M LYNN MACKENZIE \\ From the Bernhard Baron Memorial Research Laboratories, Queen Charlotte's Hospital, \\ Goldhawk Road, London W6
}

SUMMARY A technique is described for the routine preparation of seminal fluid specimens for transmission electron microscopy. The method is applicable to cases where examination of sperm morphology is required and can also be used for the indentification of non-spermatozoal cells and their inclusions.

The utility of the transmission electron microscope (TEM) in male fertility studies is becoming well recognised. Morphological abnormalities of the spermatozoon, for example the absence of dynein arms in the tail ${ }^{12}$ and the lack of an acrosomal cap in the head ${ }^{3}{ }^{4}$ can only be detected by electron microscopy. More recently the TEM has been shown to be useful in the study of non-spermatozoal cells in seminal fluid. ${ }^{5}$ One factor inhibiting the more widespread use of electron microscopy is undoubtedly the complex and time-consuming techniques required to prepare specimens. The present report describes a standardised procedure which, at least in part, overcomes this constraint by being relatively simple to perform and is equally applicable to cases of teratospermia, leucospermia and oligospermia. The procedure has been used successfully for the examination of over 200 seminal fluid specimens.

\section{Material and methods}

Two hundred and eighteen semen specimens from 190 patients attending the male subfertility clinic of the Chelsea Hospital for Women were prepared for investigation. These patients were selected on the basis of their low sperm motility or the presence of a significant number of non-spermatozoal cells in the ejaculate.

Preliminary experiments indicated that it was essential to allow the semen to liquefy before fixation to facilitate the later concentration of the cellular components. The semen specimens were fixed by adding more than an equal volume of $3 \%$ glutaraldehyde made up in $0 \cdot 1 M$ cacodylate buffer $\mathrm{pH} 7 \cdot 2-7 \cdot 4$ and $2.5 \mathrm{mM}$ calcium chloride. Fixation was for $2 \mathrm{~h}$

Accepted for publication 18 March 1981 at either $0-4^{\circ} \mathrm{C}$ or room temperature after which the specimens were centrifuged and the pellet resuspended in fresh buffer. The specimens were usually transported from the clinic to the laboratory at this stage of the procedure.

After a minimum of $24 \mathrm{~h}$ in the buffer wash, the specimens were centrifuged and post-fixed in $1 \%$ osmium tetroxide made up in $0.1 M$ cacodylate buffer $\mathrm{pH} 7 \cdot 2-7 \cdot 4$ and $2.5 \mathrm{mM}$ calcium chloride foc two hours at room temperature. The specimens were्] centrifuged and the pellet suspended and washed in $50 \%$ ethyl alcohol. After a final centrifugation the pellet was well mixed in a small volume of $50 \%$ alcohol, drawn up into a Pasteur pipette then slowly dispensed drop-wise on to the surface of a stack of fast filter papers (Whatman 41). The liquid was completely absorbed into the filter paper between drops. In this way a "pile" of cellular material was created on the surface of the filter paper (Fig. 1a), which could be removed with the aid of a fine spatula (Fig. 1b) and placed on to a 2-4\% agar plate (Oxoid Agar No 1). The material was covered with molten agar at approximately $60^{\circ} \mathrm{C}$ (Fig. 1C). After solidification surplus agar was removed from around the specimen (Fig. 1d), the "block" of material was dehydrated through a graded series of alcohols and embedded in araldite. Semithin and ultrathin sections were cut on glass knives through the maximum diameter of the $\sigma$ block. The semithin sections were stained with N toluidine blue and the ultrathin sections were $\underset{\mathrm{N}}{\mathrm{N}}$ mounted on carbon-celloidin coated copper grids, stained with uranyl acetate and lead citrate, and examined in an Hitachi HU 12A TEM operated at $75 \mathrm{kV}$.

In cases of leucospermia a differential score of leucocytes was made by counting 100 cells in a single ultrathin section. Where possible separate $\mathbb{\Phi}$ 


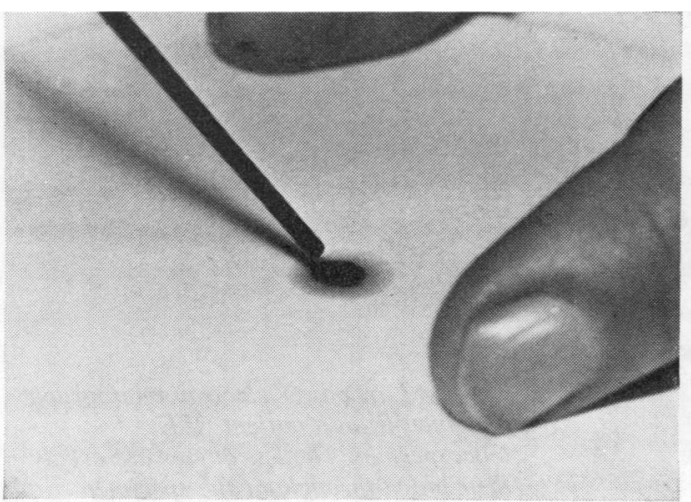

$1 \mathrm{a}$

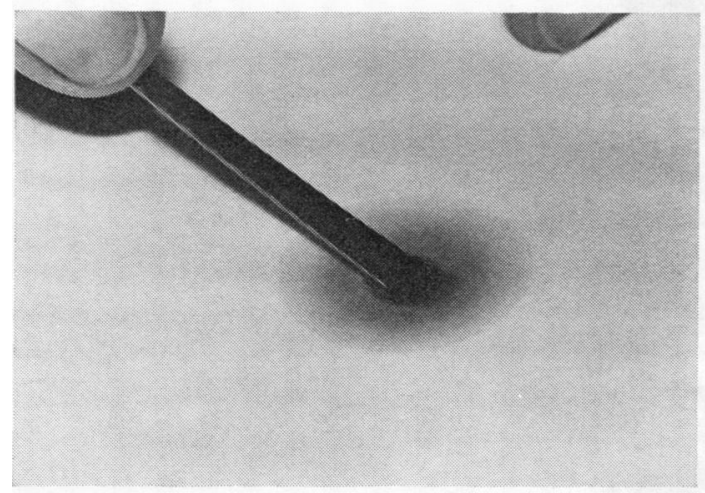

$1 \mathrm{~b}$

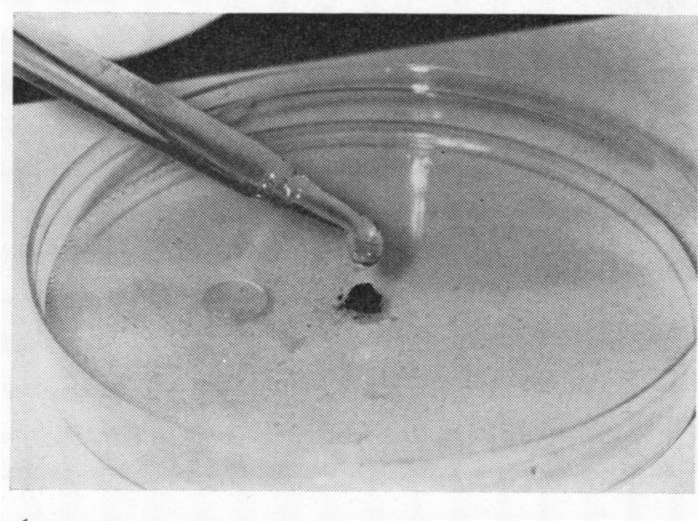

$1 \mathrm{c}$

counts were made on sections cut from different levels of the block or from alternative blocks prepared from the same specimen.

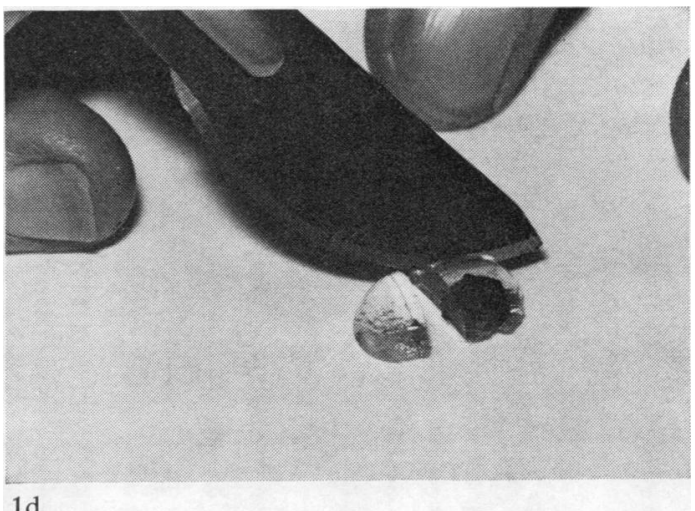

Fig. 1 Stages in the preparation of semen for electron microscopy. (a) after fixation a pile of cellular components is formed drop-wise on the surface of a fast filter paper. (b) cellular material is removed using a fine spatula. (c) once on the agar plate the specimen is covered with molten agar. (d) excess agar is trimmed away and the resulting block processed in the conventional manner.

\section{Results}

Of the 218 specimens prepared by the described technique only two proved unsuitable for examination. One had an initial mucoid consistency which prevented the concentration of the cellular components into a single pellet. It was not possible to cut ultrathin sections from the second specimen which had been collected in urine. The reason for the difficult cutting properties has not been determined.

Sections cut from the remaining 216 specimens revealed that the cellular components of the semen were both highly concentrated and also distributed evenly throughout the block (Fig. 2), there being no evidence of the stratification that results from a simple centrifugation technique. The spermatozoa were randomly orientated (Fig. 3 ) so that in any part of a section a proportion of the sperm tails were cut in transverse section (Fig. 3 inset). There were sufficient good transverse sections in a single ultrathin section for sperm tail structure to be confidently reported in 199 semen specimens, 13 of which had an initial sperm concentration of less than $5 \times 10^{6} / \mathrm{ml}$. Of the remaining 19, 3 were azoospermic, 10 had a sperm concentration of less than $1 \times 10^{6} / \mathrm{ml}$ and the remaining 6 a concentration of less than $5 \times 10^{6} / \mathrm{ml}$.

Seventy-five of the specimens contained a significant number of leucocytes in that it was possible to count at least 100 leucocytes in a single ultrathin section. To confirm that this technique produced a random distribution of cells throughout the specimen, differential counts were performed (a) in different parts of the same section and (b) in sections from 


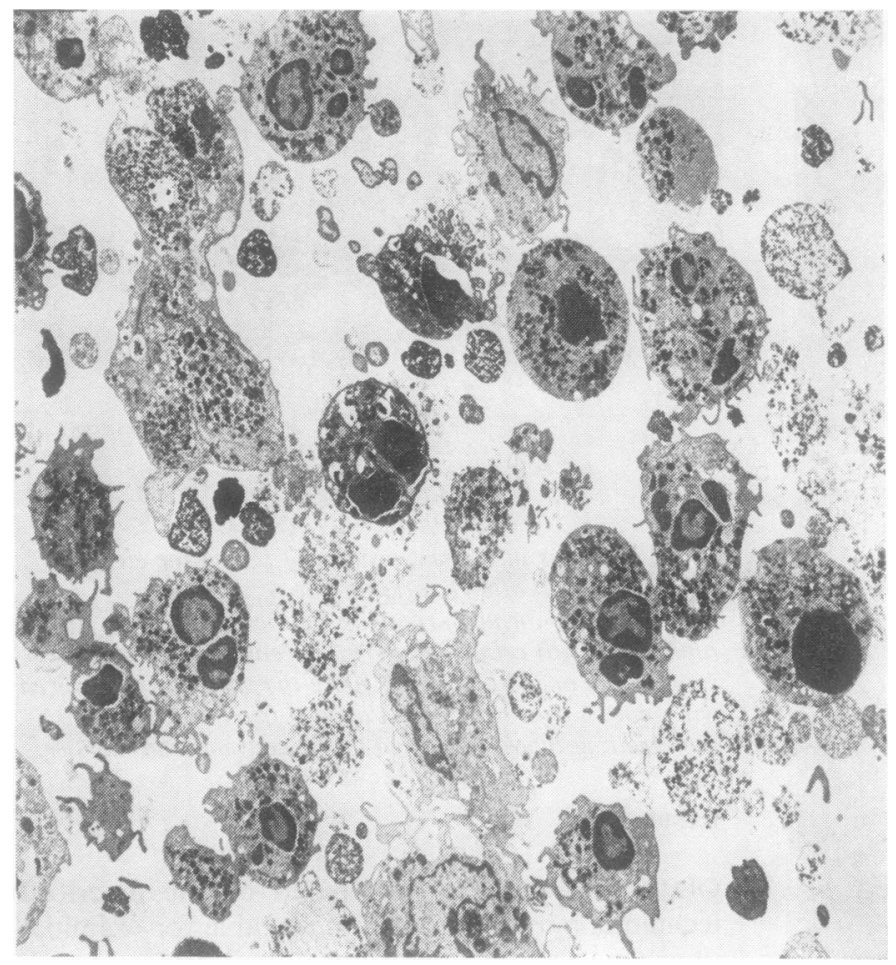

Fig. 2 Low power electron micrograph of semen from a patient with leucospermia. The leucocytes are evenly distributed throughout the specimen.

$\times 2000$.

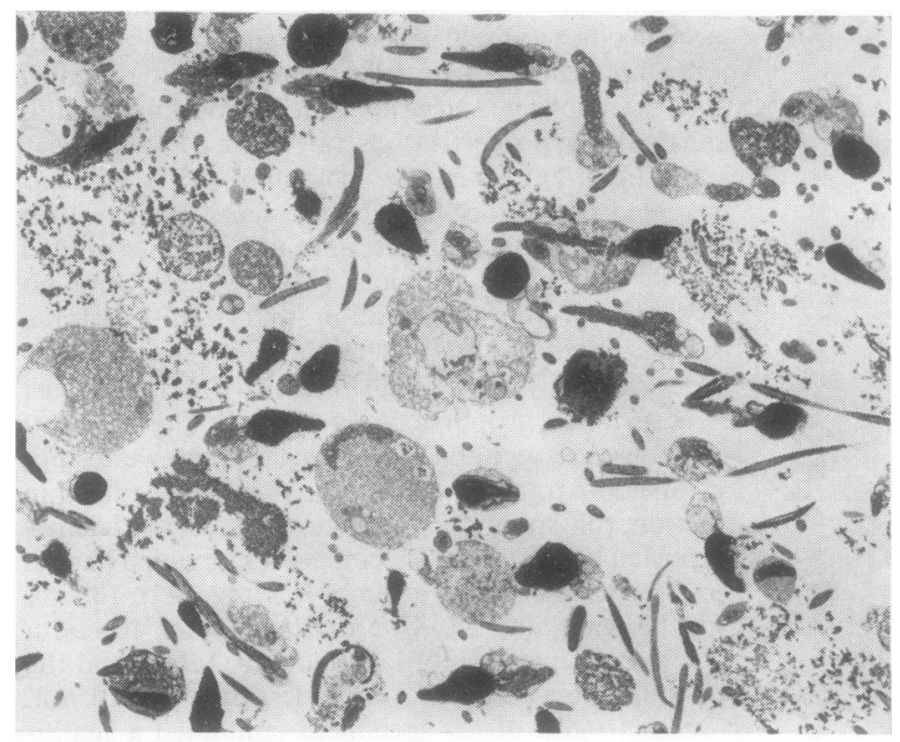

Fig. 3 Low power electron micrograph showing the random orientation of sperm within a section. $\times 2000$. below: a transverse section of a sperm tail. $\times 48000$. 
different levels of the block. In all instances the counts were reproducible to within $\pm 1 \%$. The results and clinical implications of these examinations will be presented elsewhere.

\section{Discussion}

Fine-structural studies on spermatozoa have generally employed simple centrifugation preparative techniques. Agar has been used in some instances to stabilise the pellet. ${ }^{4}$ Novikoff et al. ${ }^{6}$ have described a method using Milipore filters which avoids centrifugation while Holderegger and Bechter ${ }^{7}$ orientate sperm by squashing them between resin blocks and glass slides which facilitates the later production of longitudinal sections. None of these methods is wholly satisfactory for the routine preparation of clinical semen specimens where very low sperm counts or abundant cells are often encountered.

When the described preparative technique was devised a number of criteria were adopted. Firstly it was recognised that the technique should concentrate the spermatozoa and other cellular components. As a result it has been possible to examine sperm tail morphology of patients having very low sperm counts. Furthermore the concentration of cellular components has enabled differential cell counts to be performed on single ultrathin sections.

A random orientation of sperm within the block was obtained by introducing a mixing stage after the final centrifugation. The random orientation has greatly reduced the time and skill required for cutting ultrathin sections by comparison with techniques which attempt to maintain a fixed orientation. The lower number of transverse sections found in randomly-orientated specimens is compensated by the concentrating effect of the technique. The "mixing" of the components has the added advantage that an even distribution of non-spermatozoal cellular components is achieved. Differential counts of leucocytes made on sections cut from different parts of a block were in consistent agreement.

The method described employs a double fixation regimen of glutaraldehyde and osmium tetroxide since these are the fixatives of choice in almost all the reports on sperm morphology published in recent years. Cacodylate buffer was chosen on the basis of its comparatively long "shelf-life" which is an important consideration when introducing a method for routine clinical use. Fixation temperature and time have not been found to be critical.
All the specimens examined contained both well preserved cell and sperm structures together with highly disrupted cytoplasmic material. The differing degree of cellular preservation observed within a single specimen appears to be a feature of the selected specimens rather than reflect any shortcoming in the preparative technique. Specimens in which a general disruption of spermatozoa was noted invariably had little or no initial sperm motility. Sperm precursors showed the greatest degree of cellular disruption while leucocytes, when present, were generally well preserved. ${ }^{5}$

The lack of any excessively difficult procedure with this technique and in particular the ease with which ultrathin sections can be cut has enabled this laboratory to offer the routine electron microscopical analysis of semen specimens as a service. The results of a trial of this service with a review of the clinical advantages and implications are currently being assessed and will be reported in due course.

We wish to thank Jitka Stedronska and the staff of the male subfertility clinic, Chelsea Hospital for Women for the initial collection and prefixation of the material used in this study. We acknowledge the advice and support of Dr J Pryse-Davies, Dr Louis Hughes and Mr WF Hendry.

\section{References}

${ }^{1}$ Afzelius BA, Eliasson R, Johnsen O, Lindholmer C. Lack of dynein arms in immotile human spermatozoa. $J$ Cell Biol 1975;66:225-32.

2 Eliasson R, Mossberg B, Camner P, Afzelius BA. The immotile-cilia syndrome. N Engl J Med 1977;297:1-6.

${ }^{3}$ Pedersen M, Rebbe $H$. Fine structure of round-headed human spermatozoa. $J$ Reprod Fertil 1974;37:51-4.

${ }^{4}$ Anton-Lamprecht I, Kotzur B, Schopf E. Round-headed human spermatozoa. Fertil Steril 1976;27:685-93.

${ }^{5}$ Hughes L, Ryder TA, MacKenzie ML, Pryse-Davies J, Stedronska J, Hendry WF. The use of the transmission electron microscope to study non-spermatozoal cells in semen. In: Oligozoospermia; recent progress in andrology. Fratese G, ed. NewYork: Raven Press, 1981 :65-75.

- Novikoff PM, Cohen J, Novikoff AB, Davis C. Cytochemical visualisation of the midpieces of ejaculated human spermatozoa. J Microscopie 1971 ;11:169-74.

${ }^{7}$ Holderegger CL, Bechter R. A reliable method for obtaining longitudinal sections of sperms for electron microscopy. Experientia 1978;34:1386-7.

Requests for reprints to: Dr TA Ryder, Bernhard Baron Memorial Research Laboratories, Queen Charlotte's Hospital, Goldhawk Road, London W6, England. 